\title{
Dosing Guidelines of Aminoglycosides in Neonates: $A$ Balance Between Physiology and Feasibility
}

\author{
Karel Allegaert ${ }^{1}$, Veerle Cossey ${ }^{1}$ and John $\mathrm{N}$ van den Anker ${ }^{2,3,4,5}$
}

\begin{abstract}
${ }^{1}$ Neonatal Intensive Care Unit, University Hospitals Leuven, Belgium; ${ }^{2}$ Division of Pediatric Clinical Pharmacology, Children's National Medical Center, Washington, DC, USA and ${ }^{3}$ Departments of Pediatrics, Integrative Systems Biology, Pharmacology and Physiology, George Washington University School of Medicine and Health Sciences, Washington, DC, USA, ${ }^{4}$ Intensive Care, Erasmus MC-Sophia Children's Hospital, Rotterdam, the Netherlands, and ${ }^{5}$ Department of Paediatric Pharmacology, University Children's Hospital Basel, Switzerland
\end{abstract}

\begin{abstract}
Once daily dosing of aminoglycosides has been introduced and validated in non-neonatal patient cohorts. This is because aminoglycosides display peak concentration dependent bacterial killing, have a postantibiotic effect and adaptive resistance. In addition, this strategy reduces toxicity. Although aminoglycosides are also frequently administered to neonates, there is still debate about how to integrate and extrapolate these extended interval dosing regimens into dosing schedules tailored for neonates. There is a growing body of knowledge on aminoglycoside disposition and its covariates (e.g. asphyxia, ibuprofen or indomethacin exposure, serum creatinine, sepsis, dose accuracy) in neonates. In essence, integration of developmental physiology with clinical pharmacology unveils a discrepancy between aspects related to either body composition (higher distribution volume necessitates higher dose, to attain peak concentration) or to elimination clearance (lower renal clearance necessitates prolonged time interval between administrations).

Such discrepancy can be solved by introducing more complex dosing guidelines (based on weight, postnatal age, serum creatinine, ibuprofen, asphyxia) in neonates. However, the introduction of more complex dosing guidelines should be balanced with its clinical feasibility. At least, there are reports that these more complex dosing guidelines result in a higher incidence of dosing errors. Besides errors in prescription, these errors also relate to the number of dilutions or manipulations needed before the prescribed dose can be administered. Since an integrated approach is needed, we discuss in this overview both the available pharmacokinetic data in support of the use of extended dosing regimens in neonates as well as the strategies suggested to reduce dosing errors.
\end{abstract}

Keywords: Pharmacodynamics, pharmacokinetics, newborn, aminoglycosides, renal function, extended interval dosing, safety, care bundle.

\section{INTRODUCTION: AMINOGLYCOSIDES IN NEONATES}

Antibiotics are the most frequently administered drugs in a neonatal intensive care unit (NICU) setting, with penicillines and aminoglycosides as most commonly administered classes of antibiotics. Usually, a combination of an aminoglycoside (e.g. gentamicin, amikacin, netilmicin, tobramycin, kanamycin) and a betalactam antibiotic (e.g. penicillin G, ampicillin, amoxicillin, cephalosporins), are administered to treat suspected or proven bacterial infections in neonates. [1-4]. Aminoglycosides are traditionally administered for confirmed aerobic, Gram-negative infections (e.g. E. Coli, Enterobacter species, Klebsiella species, Pseudomonas species) or rather empirically for suspected congenital or nosocomial bacterial infections. There is a synergism with beta-lactam antibiotics for group B streptococcal and coagulase-negative staphylococcal infections. The bactericidal efficacy for gramnegative pathogens, the synergism with beta-lactam antibiotics, the limited bacterial resistance and bacterial selection pressure and the low cost contributed to this practice [5].

Despite their routine administration, most of these compounds are still administered in an off-label or unlicensed setting [6,7]. Similar to other pediatric and non-pediatric populations in whom these extended dosing interval have been validated, there is a shift towards 'once daily' or more accurate for neonates, 'extended dosing interval' approaches. This is because the primary pharmacodynamic outcome of aminoglycosides, i.e. bactericidal efficacy exclusively relates to the pharmacokinetics of the aminoglycosides, irre

*Address correspondence to this author at the Neonatal Intensive Care Unit, Division of Woman and Child, University Hospital, Herestraat 49, 3000 Leuven, Belgium; Tel: 00-32-16-343850; Fax: 00-32-16-343209;

E-mail: karel.allegaert@uz.kuleuven.ac.be spective of the characteristics of the host [5,9-12]. However, the development and introduction of such dosing regimens needs some population specific tailoring.

The combination of in vitro and in vivo bactericidal characteristics resulted in a classification of antibiotics according to their specific pharmacokinetic/pharmacodynamic (PK/PD) relationship $[1,5]$. When we focus on bacterial growth inhibition or killing, a variable concentration-response depending on this classification needs to be considered. Minimal Inhibitory Concentration (MIC) values and the most effective strategy to aim for relate to both the antibiotic and the pathogen. For instance, maintaining the serum concentration $>4$ times the MIC is the goal for beta-lactams, while reaching a peak/MIC ratio $>8$ is applied for aminoglycosides [5]. Because of differences in susceptibility of gram negative bacteria to gentamicin, tobramycin and netilmicin compared to amikacin, the equivalent concentration of amikacin is 3-4 times higher, necessitating 3-4 times higher amikacin concentrations [5].

The peak/MIC ratio relevance for aminoglycosides is because these compounds display concentration-dependent bacterial killing. Aminoglycoside mediated bacterial killing results from inhibition of bacterial protein synthesis through irreversible binding to the $30 \mathrm{~S}$ bacterial ribosome and results in deficient bacterial protein production. This uptake necessitates an active uptake process [5]. There are however additional observations that suggest that the outer bacterial membrane itself is also an important direct target for bacterial killing. Aminoglycosides induce fissures in the outer bacterial cell membrane, resulting in leakage of intracellular contents and hereby enhance subsequent (passive) antibiotic diffusion. This may in part also explain the earlier mentioned synergism with beta-lactam antibiotics, since beta-lactam antibiotics act by inhibiting the synthesis of the peptidoglycan layer of bacterial cell wall $[1,8]$. 
Based on in vitro and on extrapolation of in vivo observations in non-neonatal populations, it is generally accepted to aim for a peak aminoglycoside concentration $\left(\mathrm{C}_{\text {peak }}\right)$ to minimal inhibitory concentration (MIC) ratio $\left(\mathrm{C}_{\text {peak }} / \mathrm{MIC}\right)$ of at least 8 . Besides concentration dependent killing, there are some other issues to consider when we aim to further improve the PK/PD relation of aminoglycosides in neonates. We would like to mention the post-antibiotic effect, the concept of adaptive resistance, and the aminoglycoside exposure related toxicity (ototoxicity, nephrotoxity).

The post-antibiotic effect refers to the observation that the bacterial growth remains suppressed for at least several hours after the serum concentration of a given aminoglycoside has dropped below the pathogen specific MIC value $[1,5,8]$. Adaptive resistance refers to the observation that there is a reduction in antimicrobial killing of initially susceptible bacteria during ongoing aminoglycoside exposure. This very likely relates to bacterial membrane protein changes and altered expression of regulatory genes of the anaerobic respiratory pathway, and can be overcome with higher peak concentrations $[1,5,8]$. Very recently, a pharmacokinetic-pharmacodynamic model for gentamicin and its adaptive resistance with predictions of dosing schedules in newborn infants has been reported. The model supported an extended dosing interval of gentamicin in preterm neonates, and for all neonates, dosing intervals of 36 to 48 $\mathrm{h}$ were as effective as a 24-h dosing interval for the same total dose. However, prospective validation of such model efforts are urgently needed [11].

Besides the above mentioned issues (peak concentration, postantibiotic effect, adaptive resistance) related to more effective (i.e. bactericidal) administration of aminoglycosides, there are also observations in support of the association of extended dosing intervals and reduced aminoglycoside toxicity $[5,8]$. In contrast to the pharmacokinetics, the pharmacodynamics and toxicity of aminoglycosides are only in part unrelated to the population evaluated (e.g. bacterial resistance), since other aspects (inhibition of neuromuscular synaptic activity, nephrotoxicity, oto and vestibulo-toxicity, colonizing intestinal microbiota) may either by difficult to diagnose (neuromuscular synaptic activity, vestibulo-toxicity) in neonates or may have age-specific relevance (e.g. colonizing intestinal microbiota) [13]. Moreover, cohort studies describe associations that not necessary reflect causal relations between aminoglycoside exposure and oto- or nephrotoxicity [14].

There are two different patterns for aminoglycoside-induced ototoxicity. Ototoxicity may either result due to sustained high trough levels or is genetically determined. Several mitochondrial DNA variants in the $12 \mathrm{~S}$ rRNA gene (MT-RNR1) are associated with aminoglycoside induced hearing loss [15]. The higher toxicity is due to more effective uptake with subsequent accumulation in the inner ear hair cells with subsequent cell death merely since these mitochondrial variants are structurally more similar to the bacterial ribosomal RNA. Permanent bilateral hearing loss occurs in 0.1 to $0.3 \%$ of all live births with an increased risk in NICU graduates (1$4 \%$ ), depending on the clinical characteristics of the population reported. Neonatal hearing screening is based on auditory brain stem response (ABR), oto-acoustic emissions (OAE), or both [1621].

To illustrate the difficulty to explore the impact of ototoxic medications on hearing impairment, we refer to the cohorts evaluated by ABR reported following exposure to tobramycin $(n=625)$ or amikacin $(\mathrm{n}=615)$ [16,17]. de Hoog et al. observed failed ABR screening in 45/625 (7.2\%) while our group observed failed ABR screening in 70/615 (11\%) and confirmed hearing impairment in 25/615 (4\%). However, no independent association with aminoglycoside exposure (levels, duration) could be documented in both cohorts $[16,17]$. In the Leuven cohort, syndromal/congenital malformations (10/25) and cytomegalovirus infection (4/25) were the most relevant risk factors [17].
Cooper et al. reported on OAE screen results in 528 critically ill neonates, including both very low birth weight infants (VLBW, $<1500 \mathrm{~g}$ ) as well as more mature neonates [18]. The overall incidence of OAE screening failure was 69/528 (13.1\%) and was higher in the VLBW cohort $(29 / 85,34 \%)$, but there was only a significant association between screening failure and gentamicin exposure in the more mature group [18]. Using the same OAE assessment in 2347 neonates, Vella-Brincat et al. were unable to provide evidence of an increased risk of ototoxicity following neonatal gentamicin exposure, but described an association with vancomycin exposure [19]. Finally, using both OAE and ABR, there were no significant differences in hearing impairment when a $24 \mathrm{~h}$ gentamicin dosing regimen was compared to a $48 \mathrm{~h}$ regimen in extreme preterm neonates $(<28$ weeks) [20]. In the meta-analysis on the effectiveness and safety of extended interval dosing in neonates of Nestaas et al., data on hearing assessment were available in 210 neonates, with only one neonate with hearing impairment [21]. However, we would like to mention that there is still an need for data on long term hearing outcome or on vestibulotoxicity.

The pathogenesis of renal impairment relates to intracellular lysosomal accumulation of the aminoglycoside in the proximal renal tubular cells after glomerular filtration [22]. This accumulation leads to renal tubular cell damage and leakage of specific enzymes (e.g. retinol binding protein, $\mathrm{N}$-acetyl-beta-D-glucosaminidase, alkaline phosphatase, alanine aminopeptidase, gammaglutamyltransferase, alpha-1 microglobulin) or phospholipids in the urine [22]. This is an active but saturable transport, explaining why the median or trough instead of peak aminoglycoside level are better predictors of nephrotoxicity. Focused studies on the renal side effects of extended interval dosing of amikacin, netilmicin or gentamicin were unable to document any protective effect of extended interval dosing $[20,23,24]$. Similarly, in the meta-analysis on the effectiveness and safety of extended interval dosing in neonates of Nestaas et al., data on renal outcome were available in 589 neonates [21]. Depending on the renal biomarker used, the association of renal dysfunction and aminoglycoside exposure was common (raised serum creatinine $=30 / 40$ patients, alanine amino-peptides $=$ $20 / 20$ ) in both groups without significant differences between 'traditional dosing, $<24$ h' and 'extended interval dosing, $>24$ h' [21].

To put these renal side effects into the broader neonatal intensive care setting, we would like to mention that neither Cataldi et al. nor Vieux et al. were able to document in 172 (serum creatinine) and 269 (creatinine clearance) preterm neonates respectively that aminoglycosides had an independent significant negative effect on renal function [25-27]. However, transient renal tubular dysfunction after aminoglycosides has been described, and may warrant monitoring of electrolytes in specific cases [28]. Finally, because of the specific developmental characteristics in preterm neonates, with glomerulogenesis as an extra-uterine event up to 34 weeks of postmenstrual age, nephrotoxic drugs taken during fetal life and during postnatal nephrogenesis could interfere with nephron generation contributing to a particular magnitude of damage [29]. Such adjunctive damage could further increase the risk of renal failure in the adulthood of children born prematurely. At least, there is an need to further document long term renal outcome in former preterm neonates $[29,30]$.

In conclusion, the concepts of post antibiotic effect, adaptive resistance and the pathogenesis of aminoglycoside-induced toxicity provide evidence in support of the extended interval dosing, applied to further improve the PK/PD relation of aminoglycosides. However, convincing evidence on improved efficacy or reduced toxicity in neonates is still lacking. A higher dose results in a higher $\mathrm{C}_{\text {peak }} / \mathrm{MIC}$ ratio, adaptive resistance can be avoided due to higher $\mathrm{C}_{\text {peak }}$, the post antibiotic effect facilitates to further extend the time interval between administrations while the time interval mainly relates to prevent aminoglycoside-induced toxicity and reduction of the median concentration. 
The major challenge in neonates is however to maximize these concepts to result in a more efficacious treatment in the context of their immature distribution and excretion pathways, while still using dosing guidelines and precautions to avoid dosing errors: the balance between physiology and feasibility [31-33].

\section{COVARIATES OF AMINOGLYCOSIDE PHARMACOKI- NETICS IN NEONATES}

Drug dosing in neonates should be based on knowledge concerning the physiological characteristics of the newborn that will receive the drug, and the pharmacokinetic and-dynamic parameters of a given compound [33-35]. This means that for antibiotics, population specific estimates on pharmacokinetics and its covariates are of utmost importance to propose effective dosing regimens. It is reasonable to assume that all aminoglycosides display a similar disposition pattern and reflect the ontogeny of glomerular filtration rate $[36,37]$. For both pharmacokinetic estimates (distribution volume, clearance), we have similar maturational patterns, irrespective of the specific aminoglycoside considered, making extrapolation beyond compound specific observations feasible and relevant [37,38]. The most relevant contributors of aminoglycoside PK in neonates consequently are age, weight, and renal elimination capacity (glomerular filtration rate).

Aminoglycosides are water soluble and are unbound to plasma proteins. Consequently, the distribution volume $(\mathrm{Vd})$ reflects the water content of the body and its covariates in neonates. Aminoglycosides are primary eliminated by glomerular filtration and consequently, reflect glomerular filtration rate and its covariates in neonates [34-38]. The main driver of changes in distribution volume is the age related, maturational decrease in the proportions of extracellular body water content. Within the neonatal population, there are reports on the impact of a symptomatic patent ductus arteriosus, sepsis or the use of extracorporeal membrane oxygenation (ECMO) on this body water content [39-41]. All these covariates increase the distribution volume $(1 / \mathrm{kg})$ and consequently, result in a proportional decrease of the peak aminoglycoside concentration if dosing $(\mathrm{mg} / \mathrm{kg})$ remains unaltered.

The main drivers of changes in glomerular filtration rate in neonates are (birth)weight and age (postnatal age, postmenstrual age, gestational age). As recently described by Vieux et al. in a cohort of 269 preterm neonates, creatinine clearance relates to both gestational age and postnatal age with already a 5-fold range (7.9$\left.37.9 \mathrm{ml} / \mathrm{min} .1 .73 \mathrm{~m}^{2}\right)$ despite the limited age (27-31 weeks at birth) range at inclusion [26,27]. When extreme preterm $(<27$ weeks) or (near)term neonates are also considered, this range increases to $>10$ fold difference in aminoglycoside clearance within neonatal cohorts.

In addition to these maturational changes, disease characteristics and co-medication may further affect aminoglycoside clearance. Besides gestational and postnatal age, exposure to ibuprofen explained in part the variability in creatinine clearance observed by Vieux et al [27]. Similarly, co-administration of either ibuprofen or indomethacin results in a clinical relevant reduction in aminoglycoside clearance $[42,43]$. In Figure 1, we illustrate the impact of ibuprofen exposure on amikacin elimination half life (h) in a cohort of preterm neonates at birth [44]. Since these patients were included in a randomized, double blinded, controlled trial to document the impact of ibuprofen prophylaxis, the differences in amikacin clearance do reflect the impact of ibuprofen exposure $[44,45]$.

This phenomenon is not limited to ibuprofen, but has also been quantified for other non-steroidal anti-inflammatory drugs, including indomethacin [43]. Administration of indomethacin or ibuprofen in neonates is associated with a reduction of the glomerular filtration rate, reflected in a reduction in the elimination of drugs dependent on renal function for clearance. The impact of indomethacin co-administration on renal aminoglycoside clearance

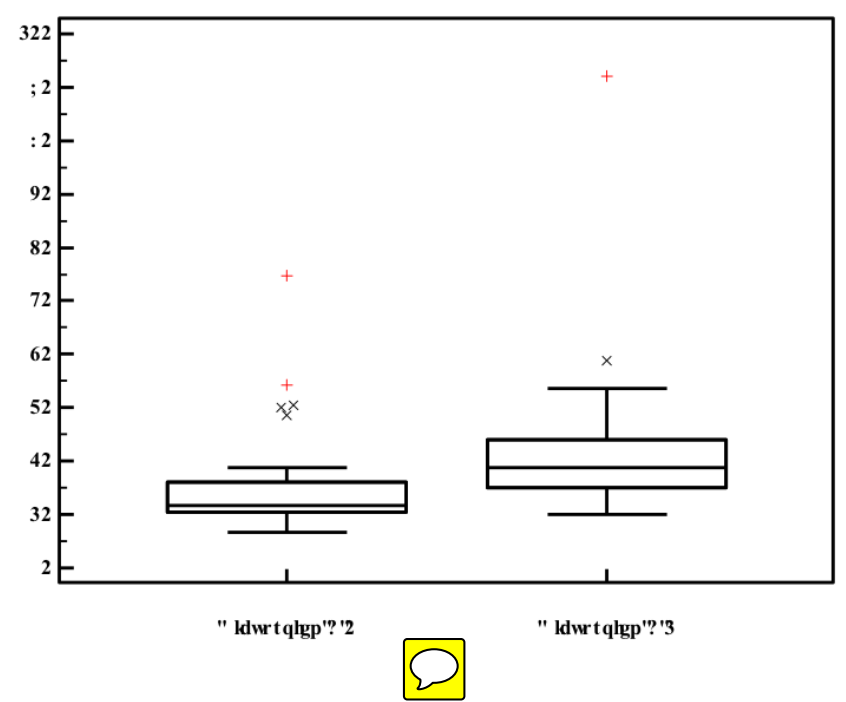

Fig. (1). Amikacin elimination serum half life (h) at birth in extreme preterm neonates with or without exposure to prophylactic ibuprofen administration, started in the first $6 \mathrm{~h}$ of postnatal life [44].

might explain the differences in trough levels observed by Thingvoll et al. versus Rastogi et al., both evaluating an extended interval dosing in very preterm neonates $[20,46]$. The incidence of gentamicin trough levels $<0.5 \mu \mathrm{g} / \mathrm{ml}$ in a $48 \mathrm{~h}$ dosing group was 30 $\%$ in the Rastogi cohort while absent in the Thingvoll cohort. Besides differences in median birth weight ( 1040 vs $743 \mathrm{~g}$ ), the Thingvoll cohort was co-treated with indomethacin as part of prophylaxis to prevent intraventricular hemorrhage [20,46]. Similar observations have been reported for netilmicin. Based on 46 treatment courses, 11 trough netilmicin levels were above the trough level aimed for $(<4 \mu \mathrm{mol} / \mathrm{l})$, of whom 7 were in their first week of life, and 5/7 co-treated with indomethacin [47]. Finally, Langhendries et al. suggested a dose extension for amikacin of $6 \mathrm{~h}$ when coexposed to indomethacin, but did not explicitly quantified the impact of indomethacin on the amikacin clearance [23]. Peripartal asphyxia is another subgroup of neonates that warrants focused attention since renal failure is commonly associated with this condition [48]. In essence, aminoglycoside clearance is reduced in these neonates but this clearance is not further affected by therapeutic hypothermia [49]. Finally, reduced clearance has also been described during ECMO [41].

In the absence of ibuprofen or indomethacin exposure, peripartal asphyxia or ECMO, it seems reasonable to predict aminoglycoside clearance based on weight and/or age (postmenstrual age, gestational age, postnatal age) $[3,4,50]$. De Cock et al. recently illustrated this based on population pharmacokinetic modelling using amikacin therapeutic drug monitoring data from 874 neonates (gestational age 24-43 weeks; postnatal age 1-30 days; birth weight 385-4650 g). The influence of different age-related, weight-related and other covariates was investigated. Postmenstrual age was the most significant covariate on clearance. However, the combination of birth weight and postnatal age proved to be superior to postmenstrual age alone, while co-administration of ibuprofen proved to be a third covariate [37]. In contrast, serum creatinine was not an independent covariate in this model. This is because serum creatinine values in neonates do not yet fully reflect the neonatal glomerular filtration rate [51-55]. Creatinaemia at birth does not yet reflect neonatal but maternal creatinine clearance and because of passive tubular back leak instead of active secretion, creatinine clearance does not yet fully reflects GFR [51-55]. Finally, absolute serum creatinine values also depend on the analytic technique used [55]. 


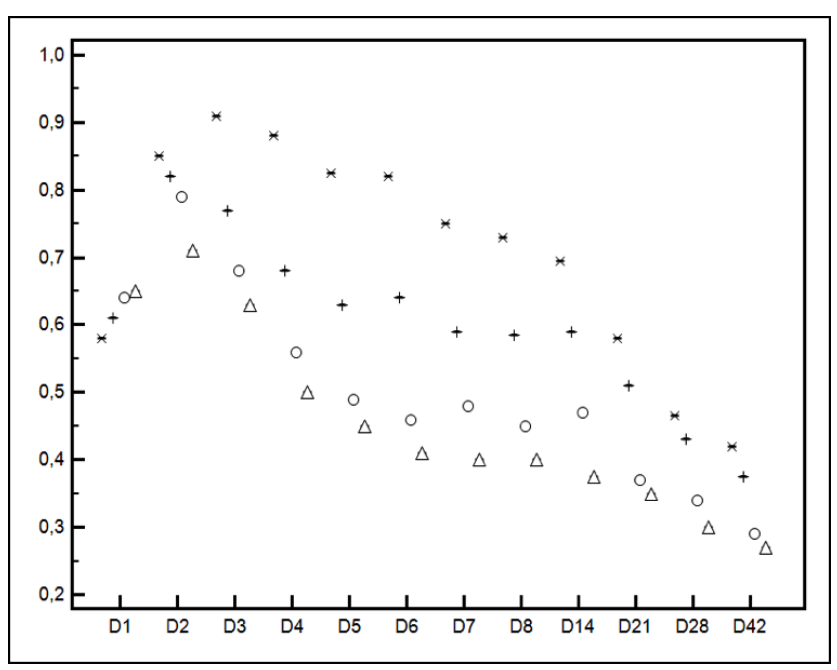

Fig. (2). Postnatal median trends of serum creatinine in neonates of various birth weight categories.

All data presented by $\mathrm{mg} / \mathrm{dl}$, all serum creatinine values were determined based on enzymatic quantification $[<1 \mathrm{~kg}(+), 1-2 \mathrm{~kg},(+), 2-3 \mathrm{~kg}(?)$ and $>3$ $\mathrm{kg}(\Delta)]$. Peak values are higher and later in the most immature cases, with a subsequent slower decrease in these neonates.

$[\mathrm{X}$-axis $=$ postnatal age, days; $\mathrm{Y}$-axis $=$ serum creatinine, $\mathrm{mg} / \mathrm{dl}]$.

In the absence of strong arguments in support of universal therapeutic drug monitoring (TDM) for aminoglycosides, it seems also reasonable to limit TDM to specific settings (e.g. peripartal asphyxia, ECMO) or prolonged administration $[3,4,50]$.

\section{FROM DEVELOPMENTAL PHYSIOLOGY TO BEDSIDE FEASIBILITY}

The integration of maturational aspects of body composition (higher distribution volume necessitates higher dose to attain peak concentration) and maturational elimination clearance (lower renal clearance necessitates longer time interval between administrations) results in more complex dosing guidelines for aminoglycosides in neonates $[4,10,11,12]$. However, this conceptual superiority should be balanced with the introduction of more complex dosing guidelines in our clinics: its feasibility needs further considerations [5662]. Studies on the feasibility and safety to implement more complex dosing guidelines are limited although there are some data on the problem of correct prescription of aminoglycosides. This feasibility includes the (in)accuracy of dosing related to multiple drug manipulations or dilutions needed before a given compound can be administered [57]. We would like to provide some illustrations on the impact of formulations used (amikacin pediatric vial) [58], the effect of product availability (netilmicin withdrawal, New Zealand) [59] and on the gentamicin guidelines (UK) [56,60,61]. Although all cases relate to aminoglycosides, these cases also serve as illustrations of the multifactorial origin of medication errors and the need of a multifactorial approach to promote safe medication practices.

Based on consecutive reports on interindividual amikacin clearance in extreme preterm $(<31$ weeks gestational age $)$ neonates at birth, we confirmed the impact of age and ibuprofen on amikacin clearance $[42,44,58]$. However, there was still unexplained variability with a clinical relevant portion of trough amikacin levels $(37 \%)$ above the desired trough level. We assumed that this unexplained variability in amikacin clearance was at least in part caused by unintended variability in dosing accuracy, inherent to any manipulation of drugs and anticipated that formulations adapted for use in neonates would result in a more accurate administration of the in- tended dose. The administration of low volumes or the need for sequential dilutions may cause additional systematic errors. Following the introduction of a pediatric vial $(50 \mathrm{mg} / \mathrm{ml}$ instead of 250 $\mathrm{mg} / \mathrm{ml}$ ), a further reduction of the interindividual variability of amikacin clearance with a reduction in the number of plasma concentrations above the desired trough level $(73 \%)$ was documented [58]. Based on a more complex population pharmacokinetic model, we confirmed that the switch form an 'adult' to 'pediatric' vial resulted in a reduction of observed variability in both distribution volume and clearance [58]. This is a strong argument in support of improved dosing accuracy following this switch. We like to present the following clinical example to further illustrate this. The dose of amikacin is 15 to $20 \mathrm{mg} / \mathrm{kg}$ in neonates. Based on the mean weight $(1078 \mathrm{~g})$ of the cohort evaluated, this means that $21.5 \mathrm{mg}$ of amikacin, equivalent to $0.085 \mathrm{ml}$ of the adult vial or $0.43 \mathrm{ml}$ of the pediatric vial should be administered. Obviously, the use of the highly concentrated vial and very low volume results in more dosing inaccuracy. This observation hereby supports the relevance of neonatal 'tailored' vials, and this clinical relevance is obviously not limited to aminoglycosides, but also includes e.g. opioids, sedatives or anti-epileptics [57]. This dosing inaccuracy is not limited to the preparation itself, since Sherwin et al. documented that the discrepancies between predicted and observed gentamicin exposure in an ex vivo model increased with smaller volumes and lower infusion rate [62]. We refer the interested reader to a recently published research protocol of a systematic review on the manipulation of drugs to obtain the required dose [27].

Besides adapted vials, unavailability or product withdrawal may affect routine practices, but potentially also efficacy and performance. Sherwin et al. reported on the impact of netilmicin withdrawal from marketing in New Zealand in 2003 [59]. The authors hereby provide evidence that the switch from netilmicin (1999$2003)$ to amikacin (2003-2007) was associated with an increase in treatment failures in the Dunedin NICU. There were differences in dosing regimen applied, in pathogens isolated and the design of the study was only observational. However, this association at least suggest that the withdrawal of a compound of which caregivers had extensive prior experience to a compound new to their practice may negative effects upon their clinical care.

Drug prescription errors are common, multifactorial and multiprofessional. Consequently, the use of a care bundle is considered to be an appropriated, structured approach for improvement of a care process. A bundle consists of a limited number of specific practices, when implemented together, result in improvement of patient outcome. Such a care bundle has been developed to improve gentamicin administration in the UK following the observation that $15 \%$ of the medication related errors related to gentamicin administration, included incorrect time, prescribing error or therapeutic drug monitoring related issues [56]. Based on these observations, the gentamicin care bundle focusses on incorrect time (the recommendation to use the 24-hour clock format) and on the need for a prompt double-check during preparation and administration without interruptions. Finally, the prescribed dose should be given within a time phrame of one hour before or after the prescribed time. In combination with a local neonatal gentamicin protocol, the assessment of compliance to this care bundle and training of the staff involved in the prescription and administration, the National Patient Safety Agency aims to reduce these medication related errors [56].

Since this Patient Safety Alert publication, there are at least two interesting observations reported that may have not been considered sufficiently, i.e. how should a dosing chart be presented, and secondly, how consistent are gentamicin dosing protocols between different units. Wong et al. recently evaluated the impact of a simplified dosing chart for gentamicin compared to the BNFc reference [60]. Errors related to the selection of the incorrect regimen, wrong frequency and one 10-fold dosing error. The pivotal difference between both charts related to the fact that in the revised version, 
the concept of postconceptional age was further elaborated to gestational + postnatal age (in weeks). It seemed that some careproviders misinterpreted 'postconceptional age', while this was no longer the case when 'gestational + postnatal age' was applied. At least, the paper re-informs us on the need to check dosing charts on the uniform interpretation.

The lack of consistence between different units has been described by Kadambari et al [61]. Based on a questionnaire analysis with answers of 43 different units, 10 different gentamicin dosing regimens were used, depending on gestational age and/or weight. Since both medical and nursing staff may rotate through different hospitals, this may result in yet another risk factor for dosing errors.

These comments bring us back to the main message of this paper. There is still debate about how to integrate and extrapolate the extended interval regimens of aminoglycosides into dosing schedules tailored for neonates. These extended interval regimens are the final result of the discrepancy between aspects related to either body composition (higher distribution volume necessitates higher dose to attain peak concentration) or to elimination clearance (lower renal clearance necessitates longer time interval between administrations). Such discrepancy can only be solved by introducing more complex dosing guidelines. However, the introduction of more complex dosing guidelines should be balanced with the clinical feasibility. Consequently, the introduction of extended dosing regimens should be supported by strategies to reduce or avoid dosing errors.

\section{CONFLICT OF INTEREST}

The authors confirm that this article content has no conflicts of interest.

\section{ACKNOWLEGDEMENTS}

Karel Allegaert is supported by the Fund for Scientific Research, Flanders (Belgium) (F.W.O. Vlaanderen) by a Fundamental Clinical Investigatorship (1800214N) gannes van den Anker is supported in part by NIH grants (R0 060543, K24DA027992, R01HD048689, U54HD071601) and FP7 grants TINN (223614), TINN2 (260908), and NEUROSIS (223060).

\section{REFERENCES}

[1] De Hoog M, Mouton JW, van den Anker JN. New dosing strategies for antibacterial agents in the neonate. Semin Fetal Neonatal Med 2005; 10: 185-94.

[2] Russell AB, Sharland M, Heath PT. Improving antibiotic prescribing in neonatal units: time to act. Arch Dis Child Fetal Neonatal Ed 2012; 97: F141-6.

[3] Van den Anker J. Use of aminoglycosides in preterm neonates: a simple task? J Pediatr 2009; 154: 935.

[4] Van den Anker JN, Allegaert K. Individualized dosing of aminoglycosides in neonates: mission accomplished or work in progress ? Eur J Clin Pharmacol 2009; 65: 1159-60.

[5] Pagkalis S, Mantadakis E, Mavros MN, Ammari C, Falagas ME. Pharmacological considerations for the proper clinical use of aminoglycosides. Drugs 2011; 71: 2277-94.

[6] d'Aloja E, Paribello F, Demontis R, Müller M. Off-label drugs prescription in neonatology: a physician's duty or a medical hazardous attitude ? J Matern Fetal Neonatal Med 2011; 24 (Suppl 1): 9

[7] Dessi A, Salemi C, Fanos V, Cuzzolin L. Drug treatments in a neonatal setting: focus on the off-label use in the first month of life. Pharm World Sci 2010; 32: 120-4.9-100.

[8] Best EJ, Gazarian M, Cohn R, Wilkinson M, et al. Once-daily gentamicin in infants and children: a prospective cohort study evaluating safety and the role of therapeutic drug monitoring in minimizing toxicity. Pediatr Infect Dis J 2011; 30: 827-32.

[9] Sherwin CM, Svahn S, Van der Linden A, Broadbent RS, Medlicott NJ, Reith DM. Individualised dosing of amikacin in neonates: a pharmacokinetic/pharmacodynamic analysis. Eur J Clin Pharmacol 2009; 65: 705-13.
[10] Allegaert K, Sherwin CM. Amikacin in neonates: dosing recommendations should be based on both pharmacokinetics and dynamics. Singapore Med J 2010; 51: 88-9.

[11] Mohamed AF, Nielsen EI, Cars O, Friberg LE. Pharmacokineticpharmacodynamic model for gentamicin and its adaptive resistance with predictions of dosing schedules in newborn infants. Antimicrob Agents Chemother 2012; 56: 179-88.

[12] Rao SC, Srinivasjois R, Hagan R, Ahmed M. One dose per day compared to multiple doses per day of gentamicin for treatment of suspected or proven sepsis in neonates. Cochrane Database Syst Rev 2011:CD005091.

[13] Langhendries JP, Maton P, François A, et al. Implementation of the intestinal micro flora in the early stage and adequate immunity later on. Arch Pediatr 2010; 17 (suppl 3): S110-8.

[14] Du W, Tutag Lehr V, Lieh-Lai M, et al. An algorithm to detect adverse drug reactions in the neonatal intensive care unit: a new approach. J Clin Pharmacol. 2013; 53: 567-73.

[15] Huth ME, Ricci AJ, Cheng AG Mechanisms of aminoglycoside ototoxicity and targets of hair cell protection. Int J Otolaryngol 2011; 2011: 937861 .

[16] De Hoog M, van Zanten BA, Hop WC, Overbosch E, WeisglasKuperus N, van den Anker JN (2003) Newborn hearing screening: tobramycin and vancomycin are not risk factors for hearing loss. $\mathrm{J}$ Pediatr 2003; 142: 41-6.

[17] Allegaert K, Kneepkens, K Vandendriessche, et al. Risk factors for abnormal newborn hearing in the era of extended dose interval amikacin administration. Basic Clin Pharmacol Toxicol 107 (Suppl 1): 171.

[18] Cooper AC, Commers AR, Finkelstein M, Lipnik PG, Tollefson LM, Wilcox RA, Hoff DS. Otoacoustic emission screen results in critically ill neonates who received gentamicin in the first week of life. Pharmacotherapy 2011; 31: 649-57.

[19] Vella-Brincat JW, Begg EJ, Robertshawe BJ, et al. Are gentamicin and/or vancomycin associated with ototoxicity in the neonate ? A retrospective audit. Neonatology 2011; 100: 186-193.

[20] Thingvoll ES, Guillet R, Caserta M, Dicenzo R. Observational trial of a 48-hour gentamicin dosing regimen derived from Monte Carlo simulations in infants born at less than 28 weeks' gestation. J Pediatr 2008; 153: 530-4

[21] Nestaas E, Bangstad HJ, Sandvik L, Wathne KO. Aminoglycoside extended interval dosing in neonates is safe and effective: a metaanalysis. Arch Dis Child Fetal Neonatal Ed 2005; 90: F294-300.

[22] Lopez-Novoa JM, Quiros Y, Vicente L, Morales AI, LopezHernandez FJ. New insights into the mechanism of aminoglycoside nephrotoxicity: an integrative point of view. Kidney Int 2011; 79: 33-45.

[23] Langhendries JP, Battisti O, Bertrand JM, et al. Adaptation in neonatology of the once-daily concept of aminoglycoside administration: evaluation of a dosing chart for amikacin in an intensive care unit. Biol Neonate 1998; 74: 351-62.

[24] Coscia A, Maiorca D, Martano C, et al. Use of netilmicin once or twice daily in preterm newborns: evaluation of nephrotoxicity by urinary alpha1-microglobulin and retinol binding protein. $\mathrm{J}$ Chemother 2008; 20: 324-6.

[25] Cataldi L, Leone R, Moretti U, et al. Potential risk factors for the development of acute renal failure in preterm newborn infants: a case-control study. Arch Dis Child Fetal Neonatal Ed 2005; 90: F514-9.

[26] Vieux R, Hascoet JM, Merdariu D, Fresson J, Guillemin F. Glomerular filtration rate reference values in very preterm children. Pediatrics 2010; 125: e1186-92.

[27] Vieux R, Desandes R, Boubred F, et al. Ibuprofen in very preterm infants impairs renal function for the first month of life. Pediatr Nephrol 2010; 25: 267-74.

[28] Giapros VI, Papadimitriou FK, Andronikou SK. Tubular disorders in low birth weight neonates after prolonged antibiotic treatment. Neonatology 2007; 91: 140-4.

[29] Schreuder MF, Bueters RR, Huigen MC, Russel FG, Masereeuw R, van den Heuvel LP. Effect of drugs on renal development. Clin J Am Soc Nephrol 2011; 6: 212-7.

[30] Jacqz-Aigrain E. Drug policy in Europe research and funding in neonates: current challenges, future perspectives, new opportunities. Early Hum Dev 2011; 87 (suppl 1): S27-S30.

[31] Fanos V, Yurdakök M. Personalized neonatal medicine. J Matern Fetal Neonatal Med 2010; ,23(Suppl 3):4-6. 
[32] Van den Anker JN. Managing drugs safely. Semin Fetal Neonatal Med 2005; 10: 73-81.

[33] Dabliz R, Levine S. Medication safety in neonates. Am J Perinatol 2012; 29: 49-56.

[34] Kearns GL, Abdel-Rahman SM, Alander SW, Blowey DL, Leeder JS, Kaufman RE. Developmental pharmacology: drug disposition, action, and therapy in infants and children. N Engl J Med 2003; 349: $1157-67$.

[35] Allegaert K, Verbesselt R, van den Anker JN, et al. Developmental pharmacology: since neonates are not just small adults. Acta Clin Belg 2008; 63: 522-30.

[36] Koren G, James A, Perlman M. A simple method for the estimation of glomerular filtration rate by gentamicin pharmacokinetics during routine drug monitoring in the newborn. 1985; Clin Pharmacol Ther 38: 680-5.

[37] De Cock RF, Allegaert K, Schreuder MF, et al. Maturation of the glomerular filtration rate in neonates, as reflected by amikacin clearance. Clin Pharmacokinet 2012; 51: 105-117.

[38] de Cock R, Piana C, Krekels EH, et al. The role of population PKPD modelling in paediatric clinical research. Eur J Clin Pharmacol 2011; 67(Suppl 1): S5-S16.

[39] van den Anker JN, Kearns GL. Gentamicin pharmacokinetics in neonates with patent ductus arteriosus. Crit Care Med 1997; 25: 1933-4.

[40] Lingvall M, Reith D, Broadbent R. The effect of sepsis upon gentamicin pharmacokinetics in neonates. Br J Clin Pharmacol 2005; 59: 54-61.

[41] Wildschut ED, Ahsman MJ, Houmes RJ, Pokorna P, de Wildt SN, Mathot RA, Tibboel D. Pharmacotherapy in neonatal and pediatric extracorporeal membrane oxygenation (ECMO). Curr Drug Metab 2012; online 22 March 2012.

[42] Allegaert K, Cossey V, Debeer A, et al. The impact of ibuprofen on renal clearance in preterm infants in independent of the gestational age. Pediatric Nephrol 2005; 20: 740-3.

[43] Fanos V, Benini D, Verlato G, Errico G, Cuzzolin L. Efficacy and renal tolerability of ibuprofen vs. indomethacin in preterm infants with patent ductus arteriosus. Fundam Clin Pharmacol 2005; 19: 187-93.

[44] Allegaert K, Cossey V, Langhendries JP, Naulaers G, Vanhole C, Devlieger H, Van Overmeire B. Effects of co-administration of ibuprofen-lysine on the pharmacokinetics of amikacin in preterm infants during the first days of life. Biol Neonate 2004; 86: 207-11.

[45] Van Overmeire B, Allegaert K, Casaer A, et al. Prohylactic ibuprofen in premature infants: a multicentre, randomised, double-blind, placebo-controlled trial. Lancet 2004; 364(9449): 1945-9.

[46] Rastogi A, Agarwal G, Pyati S, Pildes RS. Comparison of two gentamicin dosing schedules in very low birth weight infants. Pediatr Infect Dis J 2002; 21: 234-40.
[47] Berger A, Kretzer V, Gludovate P, et al. Evaluation of a netilmicinloading dose in very low birthweight infants. Biol Neonate 2003; 83: 25-9.

[48] Nouri S, Mahdhaoui N, Beizig S, et al. Acute renal failure in full term neonates with perinatal asphyxia. Prospective study of 87 cases. Arch Pediatr 2008; 15: 229-35.

[49] Liu X, Borrooah M, Stone J, Chakkarapani E, Thoresen M. Serum gentamicin concentrations in encephalopathic infants are not affected by therapeutic hypothermia. Pediatrics 2009; 124: 310-5.

[50] Young TE. Therapeutic drug monitoring - the appropriate use of drug level measurement in the care of the neonate. Clin Perinatol 2012; 39: 25-31.

[51] Bueva A, Guignard JP. Renal function in preterm neonates. Pediatr Res 1994: 36: 572-7.

[52] Rhodin MM, Anderson BJ, Peters AM, et al. Human renal function maturation: a quantitative description using weight and postmenstrual age. Pediatr Nephrol 2009; 24: 67-76.

[53] Van den Anker JN. Pharmacokinetics and renal function in the preterm infant. Acta Paediatr 1996; 85: 1393-1399.

[54] George I, Mekahli D, Rayyan M, Levtchenko E, Allegaert K. Postnatal trends in creatinemia and its covariates in extremely low birth weight (ELBW) infants. Pediatr Nephrol 2011; 26: 1843-9.

[55] Allegaert K, Kuppens M, Mekahli D, et al. Creatinine reference values in ELBW infants: impact of quantification by Jaffe or enzymatic method. J Matern Fetal Neonatal Med. 2012; 25: 1678-81.

[56] National Patient Safety Agency, NHS. Neonatal services urged to follow new gentamicin safety guidance. 15 February 2010 (www.npsa.nhs.uk/corporate/news), downloaded 26 November 2014.

[57] Richey RH, Craig JV, Shah UU, et al. The manipulation of drugs to obtain the required dose: systematic review. J Adv Nurs. 2012; 68 : 2103-12.

[58] Allegaert K, Anderson BJ, Vrancken M, et al. Impact of a paediatric vial on the magnitude of systematic medication errors in preterm neonates: amikacin as an example. Paed Perinatal Drug Ther 2006; 7: 59-63.

[59] Sherwin CM, Svahn S, Broadbent RS, Van Der Linden A, Medlicott NJ, Reith DM. Netilmicin withdrawal: impact on neonates. N Z Med J 2008; 121: 95-7.

[60] Wong E, Taylor Z, Thompson J, Tuthill D. A simplified gentamicin dosing chart is quicker and more accurate for nurse verification than the BNFc. Arch Dis Child 2009; 94: 542-5.

[61] Kadambari S, Heath PT, Sharland M, Lewis S, Nichols A, Turner MA. Variation in gentamicin and vancomcyin dosage and monitoring in UK neonatal units. J Antimicrob Chemother 2011; 66: 2647 50 .

[62] Sherwin CM, McCaffrey F, Broadbent RS, Reith DM, Medlicott NJ. Discrepancies between predicted and observed rates of intravenous gentamicin delivery for neonates. J Pharm Pharmacol 2009; 61: 465-71. 